\title{
Continuous time random walk, Mittag-Leffler waiting time and fractional diffusion: mathematical aspects
}

\author{
Rudolf GORENFLO \\ Dept. of Mathematics \& Computer Science, Freie Universität Berlin, \\ Arnimallee 3, D-14195 Berlin, Germany. \\ E-mail: gorenflo@mi.fu-berlin.de \\ Francesco MAINARDI \\ Department of Physics, University of Bologna, and INFN, \\ Via Irnerio 46, I-40126 Bologna, Italy. \\ E-mail: francesco.mainardi@unibo.it
}

Revised Version: May 2008

Invited lecture by R. Gorenflo at the 373 WE-Heraeus-Seminar on Anomalous Transport: Experimental Results and Theoretical Challenges, Physikzentrum BadHonnef (Germany), 12-16 July 2006. It will appear in the book Anomalous Transport: Foundations and Applications edited by R. Klages, G. Radons and I.M Sokolov, as Chapter 4, pp. 93-127, WILEY-VCH, Weinheim, Germany (2008).

\begin{abstract}
We show the asymptotic long-time equivalence of a generic power law waiting time distribution to the Mittag-Leffler waiting time distribution, characteristic for a time fractional continuous time random walk. This asymptotic equivalence is effected by a combination of "rescaling" time and "respeeding" the relevant renewal process followed by a passage to a limit for which we need a suitable relation between the parameters of rescaling and respeeding. As far as we know such procedure has been first applied in the 1960s by Gnedenko and Kovalenko in their theory of "thinning" a renewal process. Turning our attention to spatially one-dimensional continuous time random walks with a generic power law jump distribution, "rescaling" space can be interpreted as a second kind of "respeeding" which then, again under a proper relation between the relevant parameters leads in the limit to the space-time fractional diffusion equation. Finally, we treat the "time fractional drift" process as a properly scaled limit of the counting number of a Mittag-Leffler renewal process.
\end{abstract}




\section{Introduction}

The purpose of this paper is to outline the fundamental role the MittagLeffler function in renewal processes that are relevant in the theories of anomalous diffusion. As a matter of fact the interest in this function in statistical physics and probability theory has recently increased as is shown by the large number of papers published since 1990 of which a brief (incomplete) bibliography includes 2 , 3, 16, 21, 22, 27, 28, 29, 30, 31, 36, 41, 44, 47, 51, 60, 62, 67.

In this paper we develop a theory for long-time behaviour of a renewal process with a generic power law waiting distribution of order $\beta, 0<\beta \leq 1$ (thereby for easy readability dispensing with decoration by a slowly varying function). To bring the distant future into near sight we change the unit of time from 1 to $1 / \tau, 0<\tau \ll 1$.

For the random waiting times $T$ this means replacing $T$ by $\tau T$. Then, having very many events in a moderate span of time we compensate this compression by respeeding the whole process, actually slowing it down so that again we have a moderate number of events in a moderate span of time. We will relate the rescaling factor $\tau$ and the respeeding factor $a$ in such a way that in the limit $\tau \rightarrow 0$ we have a reasonable process, namely one whose waiting time distribution is the Mittag-Leffler waiting time distribution whose density is

$$
\phi^{M L}(t)=-\frac{d}{d t} E_{\beta}\left(-t^{\beta}\right), \quad 0<\beta \leq 1,
$$

with the Mittag-Leffler function

$$
E_{\beta}(z):=\sum_{n=0}^{\infty} \frac{z^{n}}{\Gamma(\beta n+1)}, \quad z \in \mathbf{C}, \quad \beta>0 .
$$

We will call the renewal process with waiting time density $\phi^{M L}(t)$ the Mittag-Leffler (renewal) process. This process can be seen as a fractional generalization of the Poisson process, see [40].

Our method is, in some sense, analogous to the one applied in the Sixties of the past century by Gnedenko and Kovalenko [15] in their analysis of thinning (or rarefaction) of a renewal process. They found, under certain power law assumptions, in the infinite thinning limit, for the waiting time density the Laplace transform $1 /\left(1+s^{\beta}\right)$ but did not identify it as a MittagLeffler type function. In Section 2, we provide, in our notation, an outline of 
the thinning theory for renewal processes essentially following Gnedenko and Kovalenko. Their method has inspired us for the reatment of our problems.

As we consider our renewal process formally as a continuous time random walk (CTRW) with constant non-random jumps 1 in space (for the counting function $N(t)$, in Section 3 we embed ab initio our theory into that of the CTRW, thus being in the position to treat the theory of a time fractional CTRW as limiting case of a CTRW with power law waiting time distribution. In this context the pioneering paper by Balakrishnan [1] of 1985 deserves to be mentioned. Balakrishnan already found the importance of the Laplace transform $1 /\left(1+s^{\beta}\right)$ in the time fractional CTRW and time fractional diffusion, but also did not identify it as the Laplace transform of $\phi^{M L}(t)$. Then, in 1995 Hilfer and Anton [29], see also [27, 28], showed that this waiting time density is characteristic for the time fractional CTRW and can be expressed in terms of the Mittag-Leffler function in two parameters, that is

$$
\phi^{M L}(t)=t^{\beta-1} E_{\beta, \beta}\left(-t^{\beta}\right), \quad 0<\beta \leq 1,
$$

with the generalized Mittag-Leffler function

$$
E_{\beta, \gamma}(z):=\sum_{n=0}^{\infty} \frac{z^{n}}{\Gamma(\beta n+\gamma)}, \quad z \in \mathbf{C}, \quad \beta>0, \quad \gamma \in \mathbf{R} .
$$

The form (1.3) is equivalent to the form (1.1) that we prefer as it exhibits visibly also the cumulative probability function, the survival function, $E_{\beta}\left(-t^{\beta}\right)$.

We explain in Section 4 two manipulations, rescaling and respeeding and use these in Section 5 to deduce the asymptotic universality of the MittagLeffler waiting time density under a power law assumption for the original waiting time. Then, in Section 6, assuming a suitable power law also for the spatial jumps we show that by a rescaling of the jump widths by a positive factor $h$ (that means a change of the unit of space from 1 to $1 / h$ to bring into near sight the far-away space) another respeeding is effected, now an acceleration, that in the limit $h \rightarrow 0$ (under a proper relation between $h$ and $\tau)$ leads to space-time fractional diffusion.

In Section 7, we pass to a properly scaled limit for the counting function $N(t)$ of a renewal process (again under power law assumption) and obtain the time fractional drift process (viewing $N(t)$ as a spatial variable).

We will extensively work with the transforms of Laplace and Fourier, so easing calculations and proofs of convergence in distribution (also called "weak convergence") for our passages to the limit. 
Essentially, we treat in this paper three topics. First, in Section 2, the thinning of a pure renewal process. Second, in Sections 3-6, under power law assumption for the waiting time, the asymptotic relevance of the MittagLeffler law, and then the general CTRW with special attention to space and time transition limits to fractional diffusion. As a third topic, in Section 7, we investigate the long time behaviour of the Mittag-Leffler renewal process.

Essential properties of the derivative of fractional order in time and in space are given in Appendix A and Appendix B, respectively. Finally, in Appendix $\mathrm{C}$ we give details on the two special functions of the Mittag-Leffler type that play a fundamental role in this paper, the Mittag-Leffler survival probability and the Mittag-Leffler waiting time density.

\section{An outline of the Gnedenko-Kovalenko theory of thinning}

The thinning theory for a renewal process has been considered in detail by Gnedenko and Kovalenko [15. We must note that other authors, like Szántai 63. 64 speak of rarefaction in place of thinning. Let us sketch here the essentials of this theory: in the interest of transparency and easy readability we avoid the possible decoration of the relevant power law by multiplying it with a slowly varying function. As usual we call a (measurable) positive function $a(y)$ slowly varying at zero if $a(c y) / a(y) \rightarrow 1$ with $y \rightarrow 0^{+}$for every $c>0$, slowly varying at infinity if $a(c y) / a(y) \rightarrow 1$ with $y \rightarrow+\infty$ for every $c>0$. A standard example of a slowly varying function at zero and at infinity is $|\log y|^{\gamma}$, with $\gamma \in \mathbf{R}$.

Denoting by $t_{n}, n=1,2,3, \ldots$ the time instants of events of a renewal process, assuming $0=t_{0}<t_{1}<t_{2}<t_{3}<\ldots$, with i.i.d. waiting times $T_{1}=t_{1}, T_{k}=t_{k}-t_{k-1}$ for $k \geq 2$, (generically denoted by $\mathrm{T}$ ), thinning (or rarefaction) means that for each positive index $k$ a decision is made: the event happening in the instant $t_{k}$ is deleted with probability $p$ or it is maintained with probability $q=1-p, 0<q<1$. This procedure produces a thinned or rarefied renewal process with fewer events (very few events if $q$ is near zero, the case of particular interest) in a moderate span of time.

To compensate for this loss we change the unit of time so that we still have not very few but still a moderate number of events in a moderate span of time. Such change of the unit of time is equivalent to rescaling the waiting time, multiplying it with a positive factor $\tau$ so that we have waiting times 
$\tau T_{1}, \tau T_{2}, \tau T_{3}, \ldots$, and instants $\tau t_{1}, \tau t_{2}, \tau t_{3}, \ldots$, in the rescaled process. Our intention is, vaguely speaking, to dispose on $\tau$ in relation to the rarefaction parameter $q$ in such a way that for $q$ near zero in some sense the "average" number of events per unit of time remains unchanged. In an asymptotic sense we will make these considerations precise.

Denoting by $F(t)=P(T \leq t)$ the probability distribution function of the (original) waiting time $T$, by $f(t)$ its density $(f(t)$ is a generalized function generating a probability measure) so that $F(t)=\int_{0}^{t} f\left(t^{\prime}\right) d t^{\prime}$, and analogously by $F_{k}(t)$ and $f_{k}(\mathrm{t})$ the distribution and density, respectively, of the sum of $k$ waiting times, we have recursively

$$
f_{1}(t)=f(t), \quad f_{k}(t)=\int_{0}^{t} f_{k-1}\left(t-t^{\prime}\right) d F\left(t^{\prime}\right), \text { for } k \geq 2 .
$$

Observing that after a maintained event the next one of the original process is kept with probability $q$ but dropped in favour of the second-next with probability $p q$ and, generally, $n-1$ events are dropped in favour of the $n$-th-next with probability $p^{n-1} q$, we get for the waiting time density of the thinned process the formula

$$
g_{q}(t)=\sum_{n=1}^{\infty} q p^{n-1} f_{n}(t) .
$$

With the modified waiting time $\tau T$ we have

$$
P(\tau T \leq t)=P(T \leq t / \tau)=F(t / \tau)
$$

hence the density $f(t / \tau) / \tau$, and analogously for the density of the sum of $n$ waiting times $f_{n}(t / \tau) / \tau$. The density of the waiting time of the rescaled (and thinned) process now turns out as

$$
g_{q, \tau}(t)=\sum_{n=1}^{\infty} q p^{n-1} f_{n}(t / \tau) / \tau .
$$

In the Laplace domain we have $\widetilde{f}_{n}(s)=(\widetilde{f}(s))^{n}$, hence (using $p=1-q$ )

$$
\widetilde{g}_{q}(s)=\sum_{n=1}^{\infty} q p^{n-1}(\widetilde{f}(s))^{n}=\frac{q \widetilde{f}(s)}{1-(1-q) \widetilde{f}(s)},
$$

from which by Laplace inversion we can, in principle, construct the waiting time density of the thinned process. By rescaling we get

$$
\widetilde{g}_{q, \tau}(s)=\sum_{n=1}^{\infty} q p^{n-1}(\widetilde{f}(\tau s))^{n}=\frac{q \widetilde{f}(\tau s)}{1-(1-q) \widetilde{f}(\tau s)} .
$$


Being interested in stronger and stronger thinning (infinite thinning) let us now consider a scale of processes with the parameters $\tau$ (of rescaling) and $q$ (of thinning), with $q$ tending to zero under a scaling relation $q=q(\tau)$ yet to be specified.

We have essentially two cases for the waiting time distribution: its expectation value is finite or infinite. In the first case we put

$$
\lambda=\int_{0}^{\infty} t^{\prime} f\left(t^{\prime}\right) d t^{\prime}<\infty .
$$

In the second case we assume a queue of power law type (dispensing with a possible decoration by a function slowly varying at infinity)

$$
\Psi(t):=\int_{t}^{\infty} f\left(t^{\prime}\right) d t^{\prime} \sim \frac{c}{\beta} t^{-\beta}, t \rightarrow \infty \quad \text { if } \quad 0<\beta<1,
$$

Then, by the Karamata theory (see [12, 68]) the above conditions mean in the Laplace domain

$$
\widetilde{f}(s)=1-\lambda s^{\beta}+o\left(s^{\beta}\right), \quad \text { for } \quad s \rightarrow 0^{+},
$$

with a positive coefficient $\lambda$ and $0<\beta \leq 1$. The case $\beta=1$ obviously corresponds to the situation with finite first moment $(2.6 \mathrm{a})$, whereas the case $0<\beta<1$ is related to a power law queue with $c=\lambda \Gamma(\beta+1) \sin (\beta \pi) / \pi$.

Now, passing to the limit of $q \rightarrow 0$ of infinite thinning under the scaling relation

$$
q=\lambda \tau^{\beta}, \quad 0<\beta \leq 1,
$$

between the positive parameters $q$ and $\tau$, the Laplace transform of the rescaled density $\widetilde{g_{q, \tau}}(s)$ in $(2.5)$ of the thinned process tends for fixed $s$ to

$$
\widetilde{g}(s)=\frac{1}{1+s^{\beta}},
$$

which corresponds to the Mittag-Leffler density

$$
g(t)=-\frac{d}{d t} E_{\beta}\left(-t^{\beta}\right)=\phi^{M L}(t) .
$$

Let us remark that Gnedenko and Kovalenko obtained (2.9) as the Laplace transform of the limiting density but did not identify it as the Laplace transform of a Mittag-Leffler type function. Observe that in the special case $\lambda<\infty$ we have $\beta=1$, hence as the limiting process the Poisson process, as formerly shown in 1956 by Rényi [54]. 


\section{The continuous time random walk (CTRW)}

The name continuous time random walk (CTRW) became popular in physics after Montroll, Weiss and Scher (just to cite the pioneers) in the 1960's and 1970's published a celebrated series of papers on random walks for modelling diffusion processes on lattices, see e.g. [48, 49, and the book by Weiss 66] with references therein. CTRWs are rather good and general phenomenological models for diffusion, including processes of anomalous transport, that can be understood in the framework of the classical renewal theory, as stated e.g. in the booklet by Cox [7]. In fact a CTRW can be considered as a compound renewal process (a simple renewal process with reward) or a random walk subordinated to a simple renewal process.

A spatially one-dimensional CTRW is generated by a sequence of independent identically distributed (iid) positive random waiting times $T_{1}, T_{2}, T_{3}, \ldots$, each having the same probability density function $\phi(t), t>0$, and a sequence of $i$ id random jumps $X_{1}, X_{2}, X_{3}, \ldots$, in $\mathbf{R}$, each having the same probability density $w(x), x \in \mathbf{R}$.

Let us remark that, for ease of language, we use the word density also for generalized functions in the sense of Gel'fand \& Shilov [13, that can be interpreted as probability measures. Usually the probability density functions are abbreviated by $p d f$. We recall that $\phi(t) \geq 0$ with $\int_{0}^{\infty} \phi(t) d t=$ 1 and $w(x) \geq 0$ with $\int_{-\infty}^{+\infty} w(x) d x=1$.

Setting $t_{0}=0, t_{n}=T_{1}+T_{2}+\ldots T_{n}$ for $n \in \mathbf{N}$, the wandering particle makes a jump of length $X_{n}$ in instant $t_{n}$, so that its position is $x_{0}=0$ for $0 \leq t<T_{1}=t_{1}$, and $x_{n}=X_{1}+X_{2}+\ldots X_{n}$, for $t_{n} \leq t<t_{n+1}$. We require the distribution of the waiting times and that of the jumps to be independent of each other. So, we have a compound renewal process (a renewal process with reward), compare [7].

By natural probabilistic arguments we arrive at the integral equation for the probability density $p(x, t)$ (a density with respect to the variable $x$ ) of the particle being in point $x$ at instant $t$, see e.g. [21, 23, 44, 58, 59, 60],

$$
p(x, t)=\delta(x) \Psi(t)+\int_{0}^{t} \phi\left(t-t^{\prime}\right)\left[\int_{-\infty}^{+\infty} w\left(x-x^{\prime}\right) p\left(x^{\prime}, t^{\prime}\right) d x^{\prime}\right] d t^{\prime},
$$

in which the survival function

$$
\Psi(t)=\int_{t}^{\infty} \phi\left(t^{\prime}\right) d t^{\prime}
$$


denotes the probability that at instant $t$ the particle is still sitting in its starting position $x=0$. Clearly, (3.1) satisfies the initial condition $p\left(x, 0^{+}\right)=\delta(x)$.

Note that the special choice

$$
w(x)=\delta(x-1)
$$

gives the pure renewal process, with position $x(t)=N(t)$, denoting the counting function, and with jumps all of length 1 in positive direction happening at the renewal instants.

For many purposes the integral equation (3.1) of CTRW can be easily treated by using the Laplace and Fourier transforms. Writing these as

$$
\begin{gathered}
\mathcal{L}\{f(t) ; s\}=\widetilde{f}(s):=\int_{0}^{\infty} \mathrm{e}^{-s t} f(t) d t, \\
\mathcal{F}\{g(x) ; \kappa\}=\widehat{g}(\kappa):=\int_{-\infty}^{+\infty} \mathrm{e}^{+i \kappa x} g(x) d x,
\end{gathered}
$$

then in the Laplace-Fourier domain Eq. (3.1) reads

$$
\widehat{\widetilde{p}}(\kappa, s)=\frac{1-\widetilde{\phi}(s)}{s}+\widetilde{\phi}(s) \widehat{w}(\kappa) \widehat{\widetilde{p}}(\kappa, s)
$$

Introducing formally in the Laplace domain the auxiliary function

$$
\widetilde{H}(s)=\frac{1-\widetilde{\phi}(s)}{s \widetilde{\phi}(s)}=\frac{\widetilde{\Psi}(s)}{\widetilde{\phi}(s)}, \quad \text { hence } \quad \widetilde{\phi}(s)=\frac{1}{1+s \widetilde{H}(s)},
$$

and assuming that its Laplace inverse $H(t)$ exists, we get, following [44], in the Laplace-Fourier domain the equation

$$
\widetilde{H}(s)[s \widehat{\widetilde{p}}(\kappa, s)-1]=[\widehat{w}(\kappa)-1] \widehat{\widetilde{p}}(\kappa, s),
$$

and in the space-time domain the generalized Kolmogorov-Feller equation

$$
\int_{0}^{t} H\left(t-t^{\prime}\right) \frac{\partial}{\partial t^{\prime}} p\left(x, t^{\prime}\right) d t^{\prime}=-p(x, t)+\int_{-\infty}^{+\infty} w\left(x-x^{\prime}\right) p\left(x^{\prime}, t\right) d x^{\prime},
$$

with $p(x, 0)=\delta(x)$.

If the Laplace inverse $H(t)$ of the formally introduced function $\widetilde{H}(s)$ does not exist, we can formally set $\widetilde{K}(s)=1 / \widetilde{H}(s)$ and multiply (3.6) with $\widetilde{K}(s)$. 
Then, if $K(t)$ exists, we get in place of (3.7) the alternative form of the generalized Kolmogorov-Feller equation

$$
\frac{\partial}{\partial t} p(x, t)=\int_{0}^{t} K\left(t-t^{\prime}\right)\left[-p\left(x, t^{\prime}\right)+\int_{-\infty}^{+\infty} w\left(x-x^{\prime}\right) p\left(x^{\prime}, t^{\prime}\right) d x^{\prime}\right] d t^{\prime}
$$

with $p(x, 0)=\delta(x)$.

Special choices of the memory function $H(t)$ are (i) and (ii), see eqs (3.8) and (3.12):

$$
\text { (i) } H(t)=\delta(t) \text { corresponding to } \widetilde{H}(s)=1 \text {, }
$$

giving the exponential waiting time with

$$
\widetilde{\phi}(s)=\frac{1}{1+s}, \quad \phi(t)=-\frac{d}{d t} \mathrm{e}^{-t}=\mathrm{e}^{-t}, \quad \Psi(t)=\mathrm{e}^{-t} .
$$

In this case we obtain in the Fourier- Laplace domain

$$
s \widehat{\widetilde{p}}(\kappa, s)-1=[\widehat{w}(\kappa)-1] \widehat{\widetilde{p}}(\kappa, s),
$$

and in the space-time domain the classical Kolmogorov-Feller equation

$$
\frac{\partial}{\partial t} p(x, t)=-p(x, t)+\int_{-\infty}^{+\infty} w\left(x-x^{\prime}\right) p\left(x^{\prime}, t\right) d x^{\prime}, \quad p(x, 0)=\delta(x) .
$$

(ii) $H(t)=\frac{t^{-\beta}}{\Gamma(1-\beta)}, 0<\beta<1$, corresponding to $\widetilde{H}(s)=s^{\beta-1}$,

giving the Mittag-Leffler waiting time with

$$
\widetilde{\phi}(s)=\frac{1}{1+s^{\beta}}, \quad \phi(t)=-\frac{d}{d t} E_{\beta}\left(-t^{\beta}\right)=\phi^{M L}(t), \quad \Psi(t)=E_{\beta}\left(-t^{\beta}\right) .
$$

In this case we obtain in the Fourier-Laplace domain

$$
s^{\beta-1}[s \widehat{\widetilde{p}}(\kappa, s)-1]=[\widehat{w}(\kappa)-1] \widehat{\widetilde{p}}(\kappa, s),
$$

and in the space-time domain the time fractional Kolmogorov-Feller equation

$$
{ }_{t} D_{*}^{\beta} p(x, t)=-p(x, t)+\int_{-\infty}^{+\infty} w\left(x-x^{\prime}\right) p\left(x^{\prime}, t\right) d x^{\prime}, \quad p\left(x, 0^{+}\right)=\delta(x),
$$


where ${ }_{t} D_{*}^{\beta}$ denotes the fractional derivative of of order $\beta$ in the Caputo sense, see Appendix A.

The time fractional Kolmogorov-Feller equation can be also expressed via the Riemann-Liouville fractional derivative ${ }_{t} D^{1-\beta}$, see again Appendix A, that is

$$
\frac{\partial}{\partial t} p(x, t)={ }_{t} D^{1-\beta}\left[-p(x, t)+\int_{-\infty}^{+\infty} w\left(x-x^{\prime}\right) p\left(x^{\prime}, t\right) d x^{\prime}\right],
$$

with $p\left(x, 0^{+}\right)=\delta(x)$. The equivalence of the two forms (3.15) and (3.16) is easily proved in the Fourier-Laplace domain by multiplying both sides of Eq. (3.14) with the factor $s^{1-\beta}$.

We note that the choice (i) may be considered as a limit of the choice (ii) as $\beta=1$. In fact, in this limit we find $\widetilde{H}(s) \equiv 1$ so $H(t)=t^{-1} / \Gamma(0) \equiv \delta(t)$ (according to a formal representation of the Dirac generalized function [13]), so that Eqs. (3.6)-(3.7) reduce to (3.10)-(3.11), respectively. In this case the order of the Caputo derivative reduces to 1 and that of the R-L derivative to 0 , whereas the Mittag-Leffler waiting time law reduces to the exponential.

In the sequel we will formally unite the choices (i) and (ii) by defining what we call the Mittag-Leffler memory function

$$
H^{M L}(t)= \begin{cases}\frac{t^{-\beta}}{\Gamma(1-\beta)}, & \text { if } 0<\beta<1, \\ \delta(t), & \text { if } \beta=1,\end{cases}
$$

whose Laplace transform is

$$
\widetilde{H}^{M L}(s)=s^{\beta-1}, \quad 0<\beta \leq 1 .
$$

Thus we will consider the whole range $0<\beta \leq 1$ by extending the MittagLeffler waiting time law in (3.13) to include the exponential law (3.9).

Remark: Equation (3.7) clearly may be supplemented by an arbitrary initial probability density $p(x, 0)=f(x)$. The corresponding replacement of $\delta(x)$ ) by $f(x)$ in (3.1) then requires in (3.4) multiplication of the term $(1-\widetilde{\phi}(s)) / s$ by $\widehat{f}(\kappa)$ and in (3.6) replacement of the LHS by $\widetilde{H}(s)[s \widehat{\widetilde{p}}(\kappa, s)-\widehat{f}(\kappa)]$. With $p(x, 0)=\delta(x)$ we obtain in $p(x, t)$ the fundamental solution of (3.7)

\section{Manipulations: rescaling and respeeding}

We now consider two types of manipulations on the CTRW by acting on its governing equation (3.7) in its Laplace-Fourier representation (3.6). 
(A): rescaling the waiting time, hence the whole time axis; (B): respeeding the process.

(A) means change of the unit of time (measurement). We replace the random waiting time $T$ by a waiting time $\tau T$, with the positive rescaling factor $\tau$. Our idea is to take $0<\tau \ll 1$ in order to bring into near sight the distant future. In a moderate span of time we will so have a large number of jump events. For $\tau>0$ we get the rescaled waiting time density

$$
\phi_{\tau}(t)=\phi(t / \tau) / \tau, \text { hence } \widetilde{\phi}_{\tau}(s)=\widetilde{\phi}(\tau s) .
$$

By decorating also the density $p$ with an index $\tau$ we obtain the rescaled integral equation of the CTRW in the Laplace-Fourier domain as

$$
\widetilde{H}_{\tau}(s)\left[s \widehat{\widetilde{p}}_{\tau}(\kappa, s)-1\right]=[\widehat{w}(\kappa)-1] \widehat{\widetilde{p}}_{\tau}(\kappa, s),
$$

where, in analogy to (3.5),

$$
\widetilde{H}_{\tau}(s)=\frac{1-\widetilde{\phi}(\tau s)}{s \widetilde{\phi}(\tau s)} .
$$

(B) means multiplying the quantity representing $\frac{\partial}{\partial t} p(x, t)$ by a factor $1 / a$, where $a>0$ is the respeeding factor: $a>1$ means acceleration, $0<a<1$ means deceleration. In the Laplace-Fourier representation this means multiplying the RHS of Eq. (3.6) by the factor $a$ since the expression $[s \widehat{\widetilde{p}}(\kappa, s)-1]$ corresponds to $\frac{\partial}{\partial t} p(x, t)$.

We now chose to consider the procedures of rescaling and respeeding in their combination so that the equation in the transformed domain of the rescaled and respeeded process has the form

$$
\widetilde{H}_{\tau}(s)\left[s \widehat{\widetilde{p}}_{\tau, a}(\kappa, s)-1\right]=a[\widehat{w}(\kappa)-1] \widehat{\widetilde{p}}_{\tau, a}(\kappa, s),
$$

Clearly, the two manipulations can be discussed separately: the choice $\{\tau>$ $0, a=1\}$ means pure rescaling, the choice $\{\tau=1, a>0\}$ means pure respeeding of the original process. In the special case $\tau=1$ we only respeed the original system; if $0<\tau \ll 1$ we can counteract the compression effected by rescaling to again obtain a moderate number of events in a moderate span of time by respeeding (decelerating) with $0<a \ll 1$. These vague notions will become clear as soon as we consider power law waiting times. 
Defining now

$$
\widetilde{H}_{\tau, a}(s):=\frac{\widetilde{H}_{\tau}(s)}{a}=\frac{1-\widetilde{\phi}(\tau s)}{a s \widetilde{\phi}(\tau s)} .
$$

we finally get, in analogy to (3.6), the equation

$$
\widetilde{H}_{\tau, a}(s)\left[s \widehat{\widetilde{p}}_{\tau, a}(\kappa, s)-1\right]=[\widehat{w}(\kappa)-1] \widehat{\widetilde{p}}_{\tau, a}(\kappa, s) .
$$

What is the combined effect of rescaling and respeeding on the waiting time density?

In analogy to (3.5) and taking account of (4.5) we find

$$
\widetilde{\phi}_{\tau, a}(s)=\frac{1}{1+s \widetilde{H}_{\tau, a}(s)}=\frac{1}{1+s \frac{1-\widetilde{\phi}(\tau s)}{a s \widetilde{\phi}(\tau s)}},
$$

and so, for the deformation of the waiting time density, the essential formula

$$
\widetilde{\phi}_{\tau, a}(s)=\frac{a \widetilde{\phi}(\tau s)}{1-(1-a) \widetilde{\phi}(\tau s)} .
$$

Remark: The formula (4.8) has the same structure as the thinning formula (2.5) by identification of $a$ with $q$. In both problems we have a rescaled process defined by a time scale $\tau$, and we send the relevant factors $\tau, a$ and $q$ to zero under a proper relationship. However in the thinning theory the relevant independent parameter going to 0 is that of thinning (actually respeeding) whereas in the present problem it is the rescaling parameter $\tau$.

\section{Power laws and asymptotic universality of the Mittag-Leffler waiting time density}

We have essentially two different situations for the waiting time distribution according to its first moment (the expectation value) being finite or infinite. In other words we assume for the waiting time $p d f \phi(t)$ either

$$
\rho:=\int_{0}^{\infty} t^{\prime} \phi\left(t^{\prime}\right) d t^{\prime}<\infty, \quad \text { labelled as } \beta=1,
$$

or

$$
\phi(t) \sim c t^{-(\beta+1)} \text { for } t \rightarrow \infty \text { hence } \Psi(t) \sim \frac{c}{\beta} t^{-\beta}, 0<\beta<1, c>0 .
$$


For convenience we have dispensed in (5.2) with decorating by a slowly varying function at infinity the asymptotic power law. Then, by the standard Tauberian theory (see [12, 68]) the above conditions (5.1)-(5.2) mean in the Laplace domain the (comprehensive) asymptotic form

$$
\widetilde{\phi}(s)=1-\lambda s^{\beta}+o\left(s^{\beta}\right) \quad \text { for } \quad s \rightarrow 0^{+}, \quad 0<\beta \leq 1,
$$

where we have

$$
\lambda=\rho, \quad \text { if } \quad \beta=1 ; \lambda=c \Gamma(-\beta)=\frac{c}{\Gamma(\beta+1)} \frac{\pi}{\sin (\beta \pi)}, \text { if } 0<\beta<1 .
$$

Then, fixing $s$ as required by the continuity theorem of probability theory for Laplace transforms, taking

$$
a=\lambda \tau^{\beta},
$$

and sending $\tau$ to zero, we obtain in the limit the Mittag-Leffler waiting time law. In fact, Eqs. (4.8) and (5.3) imply as $\tau \rightarrow 0$ with $0<\beta \leq 1$,

$$
\widetilde{\phi}_{\tau, \lambda \tau^{\beta}}(s)=\frac{\lambda \tau^{\beta}\left[1-\lambda \tau^{\beta} s^{\beta}+o\left(\tau^{\beta} s^{\beta}\right)\right]}{1-\left(1-\lambda \tau^{\beta}\right)\left[1-\lambda \tau^{\beta} s^{\beta}+o\left(\tau^{\beta} s^{\beta}\right)\right]} \rightarrow \frac{1}{1+s^{\beta}},
$$

the Laplace transform of $\phi^{M L}(t)$, see (1.1) and Appendix C. This formula expresses the asymptotic universality of the Mittag-Leffler waiting time law that includes the exponential law for $\beta=1$. It can easily be generalized to the case of power laws decorated with slowly varying functions, thereby using the Tauberian theory by Karamata (see again $[12,68]$ ).

Comment: The formula (5.6) says that our general power law waiting time density is gradually deformed into the Mittag-Leffler waiting time density as $\tau$ tends to zero.

Remark: Let us stress here the distinguished character of the Mittag-Leffler waiting time density $\phi^{M L}(t)=-\frac{d}{d t} E_{\beta}\left(-t^{\beta}\right)$ defined in (1.1). Considering its Laplace transform

$$
\widetilde{\phi}^{M L}(s)=\frac{1}{1+s^{\beta}}, \quad 0<\beta \leq 1,
$$

we can easily prove the identity

$$
\widetilde{\phi}_{\tau, a}^{M L}(s)=\widetilde{\phi}^{M L}\left(\tau s / a^{1 / \beta}\right) \text { for all } \tau>0, \quad a>0 .
$$


Note that Eq. (5.8) states the self-similarity of the combined operation rescaling-respeeding for the Mittag-Leffler waiting time density. In fact, (5.8) implies $\phi_{\tau, a}^{M L}(t)=\phi^{M L}(t / c) / c$ with $c=\tau / a^{1 / \beta}$, which means replacing the random waiting time $T^{M L}$ by $c T^{M L}$. As a consequences, choosing $a=\tau^{\beta}$ we have

$$
\widetilde{\phi}_{\tau, \tau^{\beta}}^{M L}(s)=\widetilde{\phi}^{M L}(s) \text { for all } \tau>0 .
$$

Hence the Mittag-Leffler waiting time density is invariant against combined rescaling with $\tau$ and respeeding with $a=\tau^{\beta}$.

Observing (5.6) we can say that $\phi^{M L}(t)$ is a $\tau \rightarrow 0$ attractor for any power law waiting time (5.2) under simultaneous rescaling with $\tau$ and respeeding with $a=\lambda \tau^{\beta}$. In other words, this attraction property of the MittagLeffler probability distribution with respect to power law waiting times (with $0<\beta \leq 1$ ) is a kind of analogy to the attraction of sums of power law jump distributions by stable distributions.

\section{Passage to the diffusion limit in space}

We have again two different situations for the jump-width distribution but according to its second moment being finite or infinite. In other words we assume for the jump-width probability density $w(x)$ (assumed for simplicity to be symmetric: $w(x)=w(-x))$ either

$$
\sigma^{2}:=\int_{-\infty}^{+\infty} x^{2} w(x) d x<\infty, \quad \text { labelled as } \alpha=2
$$

or

$$
w(x) \sim b|x|^{-(\alpha+1)} \quad \text { for } \quad|x| \rightarrow \infty, \quad 0<\alpha<2, \quad b>0 .
$$

Then we have the asymptotic relation, compare e.g. with [16, 20, 21, 22,

$$
\widehat{w}(\kappa)=1-\mu|\kappa|^{\alpha}+o\left(|\kappa|^{\alpha}\right) \quad \text { for } \quad \kappa \rightarrow 0,
$$

where

$$
\mu=\frac{\sigma^{2}}{2} \quad \text { if } \quad \alpha=2, \quad \mu=\frac{b \pi}{\Gamma(\alpha+1) \sin (\alpha \pi / 2)} \quad \text { if } \quad 0<\alpha<2,
$$

The above asymptotic relations are known in the framework of the attraction properties of the stable densities. We note that the classical book by Gnedenko and Kolmogorov [14] has unfortunately the wrong constant $\mu$ for $0<\alpha<2$. As before we dispense with the possible decoration of the relevant power law by a slowly varying function. 
By another respeeding, in fact an acceleration, we can pass over to spacetime fractional diffusion processes. For this we have three choices:

(a): diffusion limit in space only, for general waiting time,

(b): diffusion limit in space only, for ML waiting time,

(c): joint limit in time and space (with power laws in both) with scaling relation.

Note hat (b) is just a special case of (a) but of particular relevance (as we shall see). In all three cases we rescale the jump density by a factor $h>0$, replacing the random jumps $X$ by $h X$. This means changing the unit of measurement in space from 1 to $1 / h$, with $0<h \ll 1$, so bringing into near sight the far-away space. We get the rescaled jump density as $w_{h}(x)=w(x / h) / h$, corresponding to $\left.\widehat{w}_{h}(\kappa)=\widehat{w}_{(} h \kappa\right)$.

Choice (a): diffusion limit in space only, with a general waiting time law.

Starting from the Eq. (3.6), the Laplace-Fourier representation of the CTRW equation, without special assumption on the waiting time density, we fix the Fourier variable $\kappa$ and accelerate the spatially rescaled process by the respeeding factor $1 /\left(\mu h^{\alpha}\right)$, arriving at the equation (using $q_{h}$ as new dependent variable)

$$
\widetilde{H}(s)\left[s \widehat{\widetilde{q}}_{h}(\kappa, s)-1\right]=\frac{\widehat{w}(h \kappa)-1}{\mu h^{\alpha}} \widehat{\widetilde{q}}_{h}(\kappa, s) .
$$

Then, fixing $\kappa$ as required by the continuity theorem of probability theory for Fourier transforms, and sending $h$ to zero we get, noting that $[\widehat{w}(h \kappa)-$ $1] /\left(\mu h^{\alpha}\right) \rightarrow-|\kappa|^{\alpha}$, and writing $u$ in place of $q_{0}$,

$$
\widetilde{H}(s)[s \widehat{\widetilde{u}}(\kappa, s)-1]=-|\kappa|^{\alpha} \widehat{\widetilde{u}}(\kappa, s),
$$

where we still have, consistently with (3.5),

$$
\widetilde{H}(s)=\frac{1-\widetilde{\phi}(s)}{s \widetilde{\phi}(s)}=\frac{\widetilde{\Psi}(s)}{\widetilde{\phi}(s)},
$$

being $\phi(t)$ the original waiting time density. In physical space-time we have the integro-pseudo-differential equation

$$
\int_{0}^{t} H\left(t-t^{\prime}\right) \frac{\partial}{\partial t^{\prime}} u\left(x, t^{\prime}\right) d t^{\prime}={ }_{x} D_{0}^{\alpha} u(x, t), \quad 0<\alpha \leq 2,
$$

with $-|\kappa|^{\alpha}$ as the symbol of the Riesz pseudo-differential operator ${ }_{x} D_{0}^{\alpha}$ usually referred to as the Riesz fractional derivative of order $\alpha$, see Appendix B. 
Comments: By this rescaling and acceleration the jumps become smaller and smaller, their number in a given span of time larger and larger, the waiting times between jumps smaller and smaller. In the limit there are no waiting times anymore, the original waiting time density $\phi(t)$ is now only spiritual, but still determines via $H(t)$ the memory of the process. Eq. (6.7) offers a great variety of diffusion processes with memory depending on the choice of the function $H(t)$.

Choice (b): diffusion limit in space only, with a Mittag-Leffler waiting time law.

We now choose in Eq. (6.7) the Mittag-Leffler memory function (3.17), namely

$$
H(t)=H^{M L}(t), \text { hence } \widetilde{H}(s)=1 / s^{(1-\beta)},
$$

corresponding to the Mittag-Leffler waiting time law

$$
\phi^{M L}(t)=-\frac{d}{d t} E_{\beta}\left(-t^{\beta}\right), \quad 0<\beta \leq 1,
$$

consistently with the time fractional Kolmogorov-Feller equation (3.15), that includes for $\beta=1$ the classical Kolmogorov-Feller equation (3.11). As a consequence of our spatial diffusion limit, compare with [23, 44], we so arrive immediately at the space-time fractional diffusion equation

$$
{ }_{t} D_{*}^{\beta} u(x, t)={ }_{x} D_{0}^{\alpha} u(x, t), \quad 0<\alpha \leq 2,0<\beta \leq 1 .
$$

\section{Choice (c): diffusion limit combined in time and space.}

Assuming the behaviour for the waiting time density as in Eqs. (5.1)-(5.2), and for the jump-width density as in Eqs. (6.1)-(6.2), rescaling as described the waiting times and the jumps by factors $\tau$ and $h$, starting from (4.4), decelerating by a factor $\lambda \tau^{\beta}$ in time, then accelerating for space by a factor $1 /\left(\mu h^{\alpha}\right)$, we obtain (compare to Section 4 , case (B)), fixing $s$ and $\kappa$ and setting, for convenience

$$
\begin{gathered}
a(\tau, h)=\frac{\lambda \tau^{\beta}}{\mu h^{\alpha}}, \\
\widetilde{H}_{\tau}(s)\left[s \widehat{\widetilde{p}}_{\tau, a(\tau, h)}(\kappa, s)-1\right]=a(\tau, h)\left[\widehat{w}_{h}(\kappa)-1\right] \widehat{\widetilde{p}}_{\tau, a(\tau, h)}(\kappa, s),
\end{gathered}
$$

with $\widehat{w}_{h}(\kappa)=\widehat{w}(h \kappa)$ and

$$
\widetilde{H}_{\tau}(s)=\frac{1-\widetilde{\phi}(\tau s)}{s \widetilde{\phi}(\tau s)} \sim \lambda \tau^{\beta} s^{\beta-1} .
$$


Fixing $a(\tau, h)$ to the constant value 1, which means introducing the relationship of well-scaledness

$$
a(\tau, h)=\frac{\lambda \tau^{\beta}}{\mu h^{\alpha}} \equiv 1
$$

between the rescaling of time and space, we get

$$
\widetilde{H}_{\tau}(s) \sim \lambda \tau^{\beta} s^{\beta-1}, \quad \text { for } \quad \tau \rightarrow 0 .
$$

Because of

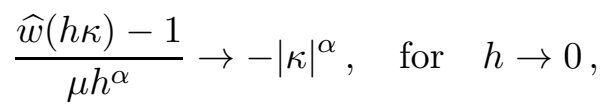

we finally get the limiting equation

$$
s^{\beta-1}[s \widehat{\widetilde{u}}(\kappa, s)-1]=-|\kappa|^{\alpha} \widehat{\widetilde{u}}(\kappa, s),
$$

corresponding to Eq. (6.8), the space-time fractional diffusion equation.

\section{Comments on some mathematical and physical aspects}

$(\alpha)$ The Mittag-Leffler waiting time (choice (b)), obeying the power law asymptotics (5.2) with $\lambda=1$ leads from (6.7) directly to the space-time fractional diffusion equation (6.8), without requirement of rescaling and deceleration in time, and with these procedures we arrive likewise at (6.8). This strange fact is caused by the invariance of the Mittag-Leffler density to the combined effects of rescaling by $\tau$ and deceleration by $\tau^{\beta}$, expressed in eq. (5.9).

$(\beta)$ Going again through our preceding deductions, we observe that the combined (well-scaled) passage of $\tau$ and $h$, under the relation (6.12), towards zero can be split in two distinct ways into two separate passages. First way: keep $h$ fixed letting $\tau$ tend to zero, then in the resulting model send also $h$ to zero. Second way: interchange the order played by $h$ and $\tau$ in the first way. Under our power law assumptions we can transform (3.7), the basic integral equation of CTRW, into Eq. (3.15) (time fractional CTRW) by rescalingrespeeding manipulation only in the time variable, and then by rescaling in space followed by an acceleration into (6.8), the space-time fractional diffusion equation. Or we can transform (3.7) by rescaling in space followed by an acceleration into Eq. (6.7) (general space fractional diffusion with memory), and then by by rescaling-respeeding in the time variable arrive at (6.8). 
$(\gamma)$ Where have the waiting times gone in the space-time fractional diffusion equation (6.8)? We can answer this question by interpreting eq. (6.10) under the scaling relation (6.12) as the Laplace-Fourier representation

$$
\widetilde{H}_{\tau}(s)\left[s \widehat{\widetilde{p}}_{\tau, 1}(\kappa, s)-1\right]=\left[\widehat{w}_{h}(\kappa)-1\right] \widehat{\widetilde{p}}_{\tau, 1}(\kappa, s),
$$

of our original CTRW (3.1), whose Laplace-Fourier representation (3.6) coincides with (6.16) if there we delete all decorations with indices. Thus eq. (6.16) represents the same physical process as (3.1) but expressed in terms of new units $1 / \tau$ and $1 / h$ of time and space, respectively. However, the respeeding factor $a(\tau, h)$ being fixed to 1 , there is no change of physical speed. When these new units are made smaller and smaller, moderate spans of time and space become numerically smaller and smaller, shrinking towards zero as $\tau$ and $h$ tend to zero, and likewise the waiting times and the jump widths shrink to zero. The distant future and the far-away space come numerically into near sight. As long as $\tau$ and $h$ are positive, we always have the same physical process, only measured in other units. The finally resulting space-time fractional diffusion process (6.8) remembers the power laws for waiting times and jumps in form of the orders $\beta$ and $\alpha$ of fractional differentiation.

$(\delta)$ An objection could be raised against the somewhat mystical actions of respeeding. Namely, if the respeeding factor $a$ in eq. (4.4) differs from 1, the underlying renewal process and consequently the whole CTRW are distorted. However, for the CTRW we carry out the actions of deceleration and acceleration in either order in succession or simultaneously in combination, and by our special choice of these factors they cancel each other in effect, so that there remains no physical distortion. This is particularly obvious in our choice (c), see the above comment $(\gamma)$.

$(\epsilon)$ Let us finally point out an advantage of splitting the passages $\tau \rightarrow 0$ and $h \rightarrow 0$. Whereas by the combined passage as in choice (c), if done in the wellscaled way (6.12), the mystical concept of respeeding can be avoided, there arises the question of correct use of the continuity theorems of probability. There is one continuity theorem for the Laplace transform, one for the Fourier transform, see [12]. Possible doubts whether their simultaneous use is legitimate vanish by applying them in succession, as in our two splitting methods.

\section{Discussion on the involved stochastic processes}

In our investigations we have met four types of spatially one-dimensional stochastic processes for the sojourn probability density $p(x, t)$ or $u(x, t)$. 
For the reader's convenience let us give a list of these processes in physical coordinates, referring to the preceding text for details, and remind briefly how they can be connected by appropriate scaling and passages to the limit. Let us note that in all these processes the initial condition $\delta(x)$ for $p\left(x, 0^{+}\right)$ or $u\left(x, 0^{+}\right)$can be replaced by a more general probability density function $f(x)$.

(I) The integral equation for the CTRW is, see (3.1) with (3.2),

$$
p(x, t)=p\left(x, 0^{+}\right) \Psi(t)+\int_{0}^{t} \phi\left(t-t^{\prime}\right)\left[\int_{-\infty}^{+\infty} w\left(x-x^{\prime}\right) p\left(x^{\prime}, t^{\prime}\right) d x^{\prime}\right] d t^{\prime}
$$

is equivalent, by the introduction of the memory function $H(t)$, see (3.5), to the generalized Kolmogorov-Feller equation, see (3.7),

$$
\int_{0}^{t} H\left(t-t^{\prime}\right) \frac{\partial}{\partial t^{\prime}} p\left(x, t^{\prime}\right) d t^{\prime}=-p(x, t)+\int_{-\infty}^{+\infty} w\left(x-x^{\prime}\right) p\left(x^{\prime}, t\right) d x^{\prime} .
$$

(II) The time fractional Kolmogorov-Feller equation, see (3.15),

$$
{ }_{t} D_{*}^{\beta} p(x, t)=-p(x, t)+\int_{-\infty}^{+\infty} w\left(x-x^{\prime}\right) p\left(x^{\prime}, t\right) d x^{\prime}, \quad 0<\beta \leq 1 .
$$

(III) The integro-pseudo-differential equation of space fractional diffusion with general memory, see (6.7),

$$
\int_{0}^{t} H\left(t-t^{\prime}\right) \frac{\partial}{\partial t^{\prime}} u\left(x, t^{\prime}\right) d t^{\prime}={ }_{x} D_{0}^{\alpha} u(x, t), \quad 0<\alpha \leq 2 .
$$

(IV) The space-time fractional diffusion equation, see (6.8),

$$
{ }_{t} D_{*}^{\beta} u(x, t)={ }_{x} D_{0}^{\alpha} u(x, t) \quad 0<\beta \leq 1, \quad 0<\alpha \leq 2 .
$$

We now sketch shortly how these four evolution equations are connected in our theory. Eq. (I) goes over in eq. (II), likewise eq. (III) in eq. (IV) by the special choice $H(t)=H^{M L}(t)$ for the memory function, see (3.17). Under our power law assumption for the waiting time, see (5.1) and (5.2), these transitions can be achieved asymptotically by manipulation via rescaling and respeeding of the underlying renewal process. Under our power law assumption for the jumps, see (6.1)and (6.2), the transition from eq. (I) to 
eq. (III) and from (II) to (IV) can be achieved asymptotically by passage to the diffusion limit only in space. Under our power law assumption for time and space there is a direct way from eq. (I) to eq. (IV), namely the well-scaled passage to the diffusion limit, for which the condition (6.12) is relevant.

\section{The time fractional drift process}

It is instructive to study the spatial transition to the diffusion limit for the Mittag-Leffler renewal process. As said in Section 3 this renewal process, viewed as a CTRW by treating its counting number $N$ as a spatial variable $x$, is obtained by choosing $w(x)=\delta(x-1)$ as the jump width density, see Eq. (3.3). Its waiting time density is, see (1.1), (3.13),

$$
\phi(t)=\phi^{M L}(t)=-\frac{d}{d t} E_{\beta}\left(-t^{\beta}\right), \quad 0<\beta \leq 1 .
$$

We have $\widetilde{H}(s)=s^{\beta-1}, \widehat{w}(\kappa)=\mathrm{e}^{i \kappa}$, hence

$$
s^{\beta-1}[s \widehat{\widetilde{p}}(\kappa, s)-1]=\left(\mathrm{e}^{i \kappa}-1\right) \widehat{\widetilde{p}}(\kappa, s) .
$$

Rescaling in space by a factor $h$ and accelerating (because of $w(\kappa)=\mathrm{e}^{i \kappa}=$ $1+i \kappa+o(\kappa)$ for $\kappa \rightarrow 0)$ this pure renewal process by the factor $1 / h$ we get a process

$$
s^{\beta-1}\left[s \widehat{\widetilde{q_{h}}}(\kappa, s)-1\right]=\frac{1}{h}\left(\mathrm{e}^{i h \kappa}-1\right) \widehat{\widetilde{q}}(\kappa, s),
$$

which as $h \rightarrow 0$ and $\kappa$ fixed gives

$$
s^{\beta-1}[s \widehat{\widetilde{u}}(\kappa, s)-1]=i \kappa \widehat{\widetilde{u}}(\kappa, s),
$$

which implies

$$
\widehat{\widetilde{u}}(\kappa, s)=\frac{s^{\beta-1}}{s^{\beta}-i \kappa} .
$$

We note that Eq. (7.2) corresponds to the time fractional drift equation

$$
{ }_{t} D_{*}^{\beta} u(x, t)=-\frac{\partial}{\partial x} u(x, t), \quad u(x, 0)=\delta(x), \quad x \in \mathbf{R}^{+}, t \in \mathbf{R}^{+} .
$$

By using the known scaling rules for the Fourier and Laplace transforms,

$$
f(a x) \stackrel{\mathcal{F}}{\leftrightarrow} a^{-1} \widehat{f}(\kappa / a), \quad a>0, \quad f(b t) \stackrel{\mathcal{L}}{\leftrightarrow} b^{-1} \widetilde{f}(s / b), \quad b>0,
$$


we infer directly from (7. 3) (thus without inverting the two transforms) the following scaling property of the (fundamental) solution

$$
u(a x, b t)=b^{-\beta} u\left(a x / b^{\beta}, t\right) .
$$

Consequently, introducing the similarity variable $x / t^{\beta}$, we can write

$$
u(x, t)=t^{-\beta} U\left(x / t^{\beta}\right),
$$

where $U(x) \equiv u(x, 1)$.

To determine the solution in the space-time domain we can follow two alternative strategies related to the different order in carrying out the inversion of the Fourier-Laplace transforms in (7. 3). Indeed we can

(S1) : invert the Fourier transform getting $\widetilde{u}(x, s)$, and then invert this Laplace transform,

(S2) : invert the Laplace transform getting $\widehat{u}(\kappa, t)$, and then invert this Fourier transform.

Strategy (S1): Recalling the Fourier transform pair,

$$
a \mathrm{e}^{-x b} \Theta(x) \stackrel{\mathcal{F}}{\leftrightarrow} \frac{a}{b-i \kappa}, \quad b>0,
$$

where $\Theta(x)$ denotes the unit step Heaviside function, we get

$$
\widetilde{u}(x, s)=s^{\beta-1} \mathrm{e}^{-x s^{\beta}} \Theta(x) .
$$

In view of the fact that $\exp \left(-s^{\beta}\right)$ is the Laplace transform of the extremal unilateral stable density of order $\beta, L_{\beta}^{-\beta}(t)$ (see for notation Appendix B), we recognize that the solution in the space-time domain can be expressed in terms of a fractional integral (see Appendix A) of such density, namely

$$
u(x, t)=\frac{1}{x^{1 / \beta}} t^{1-\beta}\left[L_{\beta}^{-\beta}\left(t / x^{1 / \beta}\right)\right] .
$$

Working in the Laplace domain we can note that the fundamental solution of our fractional drift equation (7.4) is simply related to that of the time fractional diffusion-wave equation

${ }_{t} D_{*}^{2 \beta} u(x, t)=\frac{\partial^{2}}{\partial x^{2}} u(x, t), \quad 0<\beta \leq 1, \quad x \in \mathbf{R}, t \in \mathbf{R}^{+}$,

equipped with the initial conditions $u\left(x, 0^{+}\right)=\delta(x)$ if $0<\beta \leq 1$ and $\frac{\partial}{\partial t} u\left(x, 0^{+}\right)=0$ if $1 / 2<\beta \leq 1$. In fact, the solution of (7.8) turns out the 
half of the solution (7.7) of our time fractional drift equation (7.4), extended in a symmetric way to all of $\mathbf{R}$, as can be seen by factorizing eq. (7.8) as

$$
\left({ }_{t} D_{*}^{2 \beta}-\frac{\partial^{2}}{\partial x^{2}}\right) u(x, t)=\left({ }_{t} D_{*}^{\beta}-\frac{\partial}{\partial x}\right)\left({ }_{t} D_{*}^{\beta}+\frac{\partial}{\partial x}\right) u(x, t)=0 .
$$

Indeed eq. (7.8) was solved by using the Laplace transform strategy by Mainardi in the 1990's, see e.g. [37, 38, 43] where the reader can find mathematical details of the proof and instructive plots of the fundamental solution. Then, based on Mainardi's analysis, we can state that the required solution of eq. (7.4) reads

$$
u(x, t)=t^{-\beta} M_{\beta}\left(x / t^{\beta}\right) \Theta(x),
$$

where $M$ denotes the function of Wright type defined in the complex plane

$$
M_{\beta}(z)=\sum_{n=0}^{\infty} \frac{(-z)^{n}}{n ! \Gamma[-\beta n+(1-\beta)]}=\frac{1}{\pi} \sum_{n=1}^{\infty} \frac{(-z)^{n-1}}{(n-1) !} \Gamma(\beta n) \sin (\pi \beta n) .
$$

The $M$ function is a special case of the Wright function defined by the series representation, valid in the whole complex plane,

$$
\Phi_{\lambda, \mu}(z):=\sum_{n=0}^{\infty} \frac{z^{n}}{n ! \Gamma(\lambda n+\mu)}, \quad \lambda>-1, \quad \mu \in \mathbf{C}, \quad z \in \mathbf{C} .
$$

Indeed, we recognize

$$
M_{\beta}(z)=\Phi_{-\beta, 1-\beta}(-z), \quad 0<\beta<1 .
$$

Originally, Wright introduced and investigated this function with the restriction $\lambda \geq 0$ in a series of notes starting from 1933 in the framework of the asymptotic theory of partitions. Only later, in 1940, he considered the case $-1<\lambda<0$. We note that in the handbook of the Bateman Project [10] (see Vol. 3, Ch. 18), presumably for a misprint, $\lambda$ is restricted to be non negative. For further mathematical details on the $M$-Wright function we recommend [17, 18, 24].

For our time fractional drift equation (7.4) we note the particular case $\beta=$ $1 / 2$ for which we obtain

$$
\beta=1 / 2: \quad u(x, t)=\frac{1}{\sqrt{\pi t}} \exp \left[-x^{2} /(4 t)\right], \quad x \geq 0, \quad t \geq 0 .
$$


In the limiting case $\beta=1$ we recover the rightward pure drift,

$$
\beta=1: \quad u(x, t)=\frac{1}{t} \delta(x / t-1)=\delta(x-t), \quad x \geq 0, \quad t \geq 0 .
$$

In view of the fact that that $M$-Wright function of order $\beta$ is related to the extremal unilateral stable density of order $\beta$, see [42, we conclude by displaying the alternative form of the solution of the time fractional drift equation:

$$
u(x, t)=\frac{t}{\beta x^{1+1 / \beta}} L_{\beta}^{-\beta}\left(t / x^{1 / \beta}\right),
$$

which, compared with (7.7), shows the effect of the fractional integral on the stable density function $L_{\beta}^{-\beta}$.

Strategy (S2): Recalling the Laplace transform pair, see e.g. [10, 19],

$$
E_{\beta}\left(c t^{\beta}\right) \stackrel{\mathcal{L}}{\leftrightarrow} \frac{s^{\beta-1}}{s^{\beta}-c}, \quad \Re(s)>|c|^{1 / \beta},
$$

we get

$$
\widehat{u}(\kappa, t)=E_{\beta}\left(i \kappa t^{\beta}\right)
$$

from which

$$
u(x, t)=\frac{1}{2 \pi} V P \int_{-\infty}^{+\infty} \mathrm{e}^{-i \kappa x} E_{\beta}\left(i \kappa t^{\beta}\right) d \kappa,
$$

where $V P$ denotes the Cauchy principal value. Because, see [10, Vol. 3, Chapter XVIII on Miscellaneous Functions, Section 18.1 Eq. (7),

$$
E_{\beta}(i y) \sim \frac{i}{\Gamma(1-\beta) y} \quad \text { for } y \rightarrow \pm \infty \quad \text { if } \quad 0<\beta<1,
$$

we see that $E_{\beta}(i y)$ does not tend to zero fast enough for the integral (7.17) to exist as a regular improper Riemann integral. But there should be no problem for existence as a Cauchy principal value integral. It can be shown that the present strategy based on Fourier integral (7.17) provides the result (7.9).

Remark: Not wanting to overload our paper we have deliberately avoided the concept of subordination in fractional diffusion. But, referring to [25, let us say that if in (7.15) we replace $x$ by $t_{*}$ we get the subordinator, i.e. the probability law for generating the operational time $t_{*}$ from the physical time $t$, see eq. (5.20) in [25], and, in other notation, [46]. Because of its relation (7.16) via Fourier transform to the Mittag-Leffler function with imaginary argument, the probability law governing the process (7.15) sometimes is called the Mittag-Leffler distribution, see e.g. [46]. Although so named it must not be confused with our Mittag-Leffler waiting time distribution whose density is given by (1.1). 


\section{Conclusions}

The basic role of the Mittag-Leffler waiting time probability density in time fractional continuous time random walk (CTRW) has become well known by the fundamental paper of 1995 by Hilfer and Anton [29]. Earlier in the theory of thinning (rarefaction) of a renewal process under power law assumptions, see the 1968 book by Gnedenko and Kovalenko [15], this density had been found as limit density by a combination of thinning followed by rescaling of time and imposing a proper relation between the rescaling factor and the thinning parameter. Likewise one arrives at this law when wanting to construct a certain special class of anomalous random walks, see the 1985 paper by Balakrishnan [1, the anomaly defined by growth of the second moment of the sojourn probability density like a power of time with exponent between 0 and 1. Balakrishnan's paper, having appeared a few years before the fundamental paper of 1989 by Schneider and Wyss [61], is difficult to read as it is written in a style different from the present one, so we will here not go into details. But let it be said that by well-scaled passage to the limit from CTRW (again under suitable power law assumptions in space and time) he obtained the space-time fractional diffusion equation in form of an equivalent integro-differential equation. Unfortunately, Balakrishnan's paper did not find the attention it would have deserved. However, due to the sad fact that the Mittag-Leffler function too long played a rather neglected role in treatises on special functions Balakrishnan as well as Gnedenko and Kovalenko contented themselves with presenting their results only in the Laplace transform domain; they did not identify their limit density as a Mittag-Leffler type function.

Having worked ourselves for some time on questions of well-scaled passage to the diffusion limit from continuous time random walks to fractional diffusion, see $[21,22,23,26,45,60$, we got from the theory of thinning the idea that it should be possible to carry out the passages to the limit separately in space and in time. In time this can be done by a combination of re-scaling time and respeeding the underlying renewal process (formally treating it as a CTRW with unit steps in space). In fact, thinning in the sense of Gnedenko and Kovalenko transforms the original renewal process into one that is running more slowly and this effect can be balanced by proper choice of the rescaling factor. The result of our combination of rescaling and respeeding for a CTRW governed by a given renewal process with a generic power law waiting time law is a time fractional CTRW. By another rescaling in space (now under power law assumption for the jumps) which can be interpreted as a second respeeding we arrive at the already classical space-time fractional 
diffusion equation. In this way we shed new light on the long time and wide space behaviour of continuous time random walks.

In a series of comments at the end of Section 6 , we have explained how, by what we call well-scaled passage to the diffusion limit, the transition from the CTRW to the space-time fractional diffusion process actually can be obtained by merely rescaling time and space without any respeeding at all. However, the separate passages to the limit are more satisfying with respect to mathematical rigour.

Finally, in Section 7, we have treated the time fractional drift process as a properly scaled limit of the counting function of a pure renewal process governed by a waiting time law of Mittag-Leffler type. Our trick in finding the limiting waiting time law of this renewal process consists in treating it as a CTRW with positive jumps of size 1 so that its counting number acts as a spatial variable. Then, by suitably rescaling this spatial variable, we obtain as an interesting side result the long time behaviour of the Mittag-Leffler renewal process.

\section{Appendix A: The time fractional derivatives}

For a sufficiently well-behaved function $f(t)(t \geq 0)$ we define the Caputo time fractional derivative of order $\beta$ with $0<\beta<1$ through

$$
\mathcal{L}\left\{{ }_{t} D_{*}^{\beta} f(t) ; s\right\}=s^{\beta} \widetilde{f}(s)-s^{\beta-1} f\left(0^{+}\right), \quad f\left(0^{+}\right):=\lim _{t \rightarrow 0^{+}} f(t),
$$

so that

$$
{ }_{t} D_{*}^{\beta} f(t):=\frac{1}{\Gamma(1-\beta)} \int_{0}^{t} \frac{f^{\prime}(\tau)}{(t-\tau)^{\beta}} d \tau, \quad 0<\beta<1 .
$$

Such operator has been referred to as the Caputo fractional derivative since it was introduced by Caputo in the late 1960's for modelling the energy dissipation in the rheology of the Earth, see [4, 5]. Soon later this derivative was adopted by Caputo and Mainardi in the framework of the linear theory of viscoelasticity, see [6].

The reader should observe that the Caputo fractional derivative differs from the usual Riemann-Liouville (R-L) fractional derivative

$$
{ }_{t} D^{\beta} f(t):=\frac{d}{d t}\left[\frac{1}{\Gamma(1-\beta)} \int_{0}^{t} \frac{f(\tau) d \tau}{(t-\tau)^{\beta}}\right], \quad 0<\beta<1 .
$$


Both derivatives are related to the Riemann Liouville (R-L) fractional integral that is defined for any order $\beta>0$ as

$$
{ }_{t} J^{\beta} f(t):=\frac{1}{\Gamma(\beta)} \int_{0}^{t} \frac{f(\tau) d \tau}{(t-\tau)^{1-\beta}}, \quad \beta>0,
$$

so that $\mathcal{L}\left\{{ }_{t} J^{\beta} f(t) ; s\right\}=s^{-\beta} \tilde{f}(s)$. Incidentally ${ }_{t} J^{\alpha}{ }_{t} J^{\beta}={ }_{t} J^{\alpha+\beta}$ for $\alpha, \beta>$ 0 . Then, in virtue of eqs (A.2)-(A.4), the two fractional derivatives read:

$$
\begin{array}{ll}
{ }_{t} D^{\beta}:={ }_{t} D^{1}{ }_{t} J^{1-\beta}, & 0<\beta<1, \\
{ }_{t} D_{*}^{\beta}:={ }_{t} J^{1-\beta}{ }_{t} D^{1}, & 0<\beta<1 .
\end{array}
$$

In particular, the R-L derivative of order $\beta$ is the left inverse of the corresponding R-L fractional integral in that ${ }_{t} D^{\beta}{ }_{t} J^{\beta} f(t)=f(t)$.

We note the relationships between the two fractional derivatives (when both of them exist), for $0<\beta<1$,

$$
{ }_{t} D_{*}^{\beta} f(t)={ }_{t} D^{\beta}\left[f(t)-f\left(0^{+}\right)\right]={ }_{t} D^{\beta} f(t)-\frac{t^{-\beta}}{\Gamma(1-\beta)} f\left(0^{+}\right) .
$$

As a consequence we can interpret the Caputo derivative as a sort of regularization of the $\mathrm{R}-\mathrm{L}$ derivative as soon as $f\left(0^{+}\right)$is finite; in this sense such fractional derivative was independently introduced in 1968 by Dzherbashyan and Nersesian [9], as pointed out by Kochubei, see [33, 34. In this respect the regularized fractional derivative is sometimes referred to as the Caputo-Dzherbashyan derivative.

We observe the different behaviour of the two fractional derivatives (A.2), (A.3) at the end points of the parameter interval $(0,1)$, as it can be noted from their definitions in operational terms (A.5), (A.6). In fact, whereas for $\beta \rightarrow 1^{-}$both derivatives reduce to ${ }_{t} D^{1}$, due to the fact that the operator ${ }_{t} J^{0}=I$ commutes with ${ }_{t} D^{1}$, for $\beta \rightarrow 0^{+}$we have

$$
\beta \rightarrow 0^{+} \Longrightarrow\left\{\begin{array}{l}
{ }_{t} D^{\beta} f(t) \rightarrow{ }_{t} D^{1}{ }_{t} J^{1} f(t)=f(t), \\
{ }_{t} D_{*}^{\beta} f(t) \rightarrow{ }_{t} J^{1}{ }_{t} D^{1} f(t)=f(t)-f\left(0^{+}\right) .
\end{array}\right.
$$

The above behaviours have induced us to keep for the Riemann-Liouville derivative the same symbolic notation as for the standard derivative of integer order, while for the Caputo derivative to decorate the corresponding symbol with subscript $*$.

For the R-L derivative the Laplace transform reads for $0<\beta<1$

$\mathcal{L}\left\{{ }_{t} D^{\beta} f(t) ; s\right\}=s^{\beta} \widetilde{f}(s)-g\left(0^{+}\right), g\left(0^{+}\right)=\lim _{t \rightarrow 0^{+}} g(t), g(t):={ }_{t} J^{(1-\beta)} f(t)$. 
Thus the rule (A.9) is more cumbersome to be used than (A.1) since it requires the initial value of an extra function $g(t)$ related to the given $f(t)$ through a fractional integral. However, when $\left.f^{(} 0^{+}\right)$is finite we recognize $g\left(0^{+}\right)=0$.

In the limit $\beta \rightarrow 1^{-}$both derivatives reduce to the derivative of the first order so we recover the corresponding standard formula for the Laplace transform:

$$
\mathcal{L}\left\{{ }_{t} D^{1} f(t) ; s\right\}=s \widetilde{f}(s)-f\left(0^{+}\right) .
$$

We conclude this Appendix noting that in a proper way both derivatives can be generalized for any order $\beta>1$, see e.g. [19, 53].

\section{Appendix B: The space fractional derivatives}

Let us first recall that a generic linear pseudo-differential operator $A$, acting with respect to the variable $x \in \mathbf{R}$, is defined through its Fourier representation, namely

$$
\mathcal{F}\{A f(x) ; \kappa\}:=\int_{-\infty}^{+\infty} \mathrm{e}^{i \kappa x} A f(x) d x=\widehat{A}(\kappa) \widehat{f}(\kappa), \quad \kappa \in \mathbf{R}
$$

where $\widehat{A}(\kappa)$ is referred to as the symbol of $A$, formally given as

$$
\widehat{A}(\kappa)=\left(A \mathrm{e}^{-i \kappa x}\right) \mathrm{e}^{+i \kappa x} .
$$

The fractional Riesz derivative ${ }_{x} D_{0}^{\alpha}$ is defined as the pseudo-differential operator with symbol $-|\kappa|^{\alpha}$. This means that for a sufficiently well-behaved (generalized) function $f(x)(x \in \mathbf{R})$ we have

$$
\mathcal{F}\left\{{ }_{x} D_{0}^{\alpha} f(x) ; \kappa\right\}=-|\kappa|^{\alpha} \widehat{f}(\kappa), \quad \kappa \in \mathbf{R} .
$$

The symbol of the Riesz fractional derivative is nothing but the logarithm of the characteristic function of the generic symmetric stable (in the Lévy sense) probability density, see [11, 12, 57]. Noting $-|\kappa|^{\alpha}=-\left(\kappa^{2}\right)^{\alpha / 2}$, we recognize that

$$
{ }_{x} D_{0}^{\alpha}=-\left(-\frac{d^{2}}{d x^{2}}\right)^{\alpha / 2} .
$$

In other words, the Riesz derivative is a symmetric fractional generalization of the second derivative to orders less than 2 . In an explicit way the Riesz 
derivative reads, for $0<\alpha<2$,

$$
\begin{aligned}
{ }_{x} D_{0}^{\alpha} \quad & f(x)=\frac{d^{\alpha}}{d|x|^{\alpha}} f(x) \\
& =\Gamma(1+\alpha) \frac{\sin (\alpha \pi / 2)}{\pi} \int_{0}^{\infty} \frac{f(x+\xi)-2 f(x)+f(x-\xi)}{\xi^{1+\alpha}} d \xi,
\end{aligned}
$$

where in the L.H.S we have also adopted the alternative and illuminating notation introduced by Zaslavsky, see e.g. [56]. This operator is referred to as the Riesz fractional derivative since it is obtained from the inversion of the fractional integral originally introduced by Marcel Riesz in the late 1940's, known as the Riesz potential, see e.g. [57]. It is based on a suitable regularization of a hyper-singular integral, according to a method formerly introduced by Marchaud in 1927.

Remark: Straightforward generalization to the Riesz-Feller derivative of order $\alpha$ and skewness $\theta$ is possible. Such pseudo-differential operator is denoted by us as

$$
{ }_{x} D_{\theta}^{\alpha}, \quad \text { with } \quad 0<\alpha \leq 2, \quad \theta \in \mathbf{R}, \quad|\theta| \leq \min \{\alpha, 2-\alpha\} .
$$

In this case we have

$$
\mathcal{F}\left\{{ }_{x} D_{\theta}^{\alpha} f(x) ; \kappa\right\}=-|\kappa|^{\alpha} i^{\theta \operatorname{sign} \kappa} \widehat{f}(\kappa) \quad \kappa \in \mathbf{R} .
$$

In an explicit way the Riesz-Feller derivative reads, for $0<\alpha<2$,

$$
\begin{aligned}
{ }_{x} D_{\theta}^{\alpha} f(x) & =\frac{\Gamma(1+\alpha)}{\pi}\left\{\sin [(\alpha+\theta) \pi / 2] \int_{0}^{\infty} \frac{f(x+\xi)-f(x)}{\xi^{1+\alpha}} d \xi\right. \\
& \left.+\sin [(\alpha-\theta) \pi / 2] \int_{0}^{\infty} \frac{f(x-\xi)-f(x)}{\xi^{1+\alpha}} d \xi\right\} .
\end{aligned}
$$

Notice in (B.7) that $i^{\theta \operatorname{sign} \kappa}=\exp [i(\operatorname{sign} \kappa) \theta \pi / 2]$. Thus the symbol of the Riesz-Feller fractional derivative is the logarithm of the characteristic function of the more general (strictly) stable probability density, closely following the Feller parameterization, see [11, 12] revisited by the present authors in 20]. According to our notation, the strictly stable density of order $\alpha$ and skewness $\theta$ is denoted by $L_{\alpha}^{\theta}(x)$. We note that the allowed region for the parameters $\alpha$ and $\theta$ turns out to be a diamond in the plane $\{\alpha, \theta\}$ with vertices in the points $(0,0),(1,1),(2,0),(1,-1)$, that we call the Feller-Takayasu diamond, see Fig. 1. For more details we refer the reader to [42, where series representations and numerical plots of the stable densities $L_{\alpha}^{\theta}(x)$ are found. In particular, we recall that the extremal stable densities obtained for $\theta= \pm \alpha$ with $0<\alpha<1$ are unilateral, with support in $\mathbf{R}^{\mp}$, respectively. 


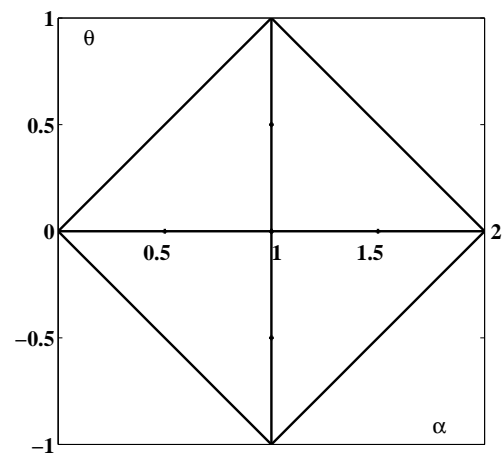

Fig. 1 The Feller-Takayasu diamond

\section{Appendix C: The Mittag-Leffler function}

The Mittag-Leffler function with parameter $\beta$ is defined as

$$
E_{\beta}(z):=\sum_{n=0}^{\infty} \frac{z^{n}}{\Gamma(\beta n+1)}, \quad \beta>0, \quad z \in \mathbf{C} .
$$

It is an entire function of order $\beta$ and reduces for $\beta=1$ to $\exp (z)$. For detailed information on the functions of Mittag-Leffler type the reader may consult e.g. [10, 19, 32, 39, 53, 57] and references therein.

Hereafter, we find it convenient to summarize the most relevant features of the functions

$$
\begin{gathered}
\Psi(t):=E_{\beta}\left(-t^{\beta}\right), \quad 0<\beta<1, \\
\phi(t):=-\frac{d}{d t} E_{\beta}\left(-t^{\beta}\right), \quad 0<\beta<1,
\end{gathered}
$$

that turn out to be the most relevant functions of Mittag-Leffler type for our purposes. Both of them reduce to the exponential function $\exp (-t)$ in the limit as $\beta \rightarrow 1$.

We begin to quote their expansions in power series of $t^{\beta}$ (convergent for $t \geq 0)$ and their asymptotic representations for $t \rightarrow \infty$,

$$
\begin{gathered}
\Psi(t)=\sum_{n=0}^{\infty}(-1)^{n} \frac{t^{\beta n}}{\Gamma(\beta n+1)} \sim \frac{\sin (\beta \pi)}{\pi} \frac{\Gamma(\beta)}{t^{\beta}}, \\
\phi(t)=\frac{1}{t^{1-\beta}} \sum_{n=0}^{\infty}(-1)^{n} \frac{t^{\beta n}}{\Gamma(\beta n+\beta)} \sim \frac{\sin (\beta \pi)}{\pi} \frac{\Gamma(\beta+1)}{t^{\beta+1}} .
\end{gathered}
$$


The Laplace transforms of $\Psi(t)$ and $\phi(t)$ can easily be obtained by transforming the series (C.4), (C.5) term by term, respectively: they read

$$
\widetilde{\Psi}(s)=\frac{s^{\beta-1}}{1+s^{\beta}}, \quad \widetilde{\phi}(s)=\frac{1}{1+s^{\beta}}, \quad \Re s>0 .
$$

For $0<\beta<1$ both functions $\Psi(t), \phi(t)$ keep the complete monotonicity of the limiting exponential function of $\exp (-t)$. Complete monotonicity of a function $f(t)$ means, for $n=0,1,2, \ldots$, and $t \geq 0,(-1)^{n} \frac{d^{n}}{d t^{n}} f(t) \geq 0$, or equivalently, its representability as (real) Laplace transform of a nonnegative function or measure, see e.g. [12].

Recalling the theory of the Mittag-Leffler functions of order less than 1, we obtain for $0<\beta<1$ the following representations, see e.g. [19],

$$
\begin{aligned}
& \Psi(t)=\frac{\sin (\beta \pi)}{\pi} \int_{0}^{\infty} \frac{r^{\beta-1} \mathrm{e}^{-r t}}{r^{2 \beta}+2 r^{\beta} \cos (\beta \pi)+1} d r, \quad t \geq 0, \\
& \phi(t)=\frac{\sin (\beta \pi)}{\pi} \int_{0}^{\infty} \frac{r^{\beta} \mathrm{e}^{-r t}}{r^{2 \beta}+2 r^{\beta} \cos (\beta \pi)+1} d r, \quad t \geq 0 .
\end{aligned}
$$

In Figs 2 and 3 we exhibit plots of the functions $\Psi(t)$ and $\phi(t)$, respectively in logarithmic and linear scales.
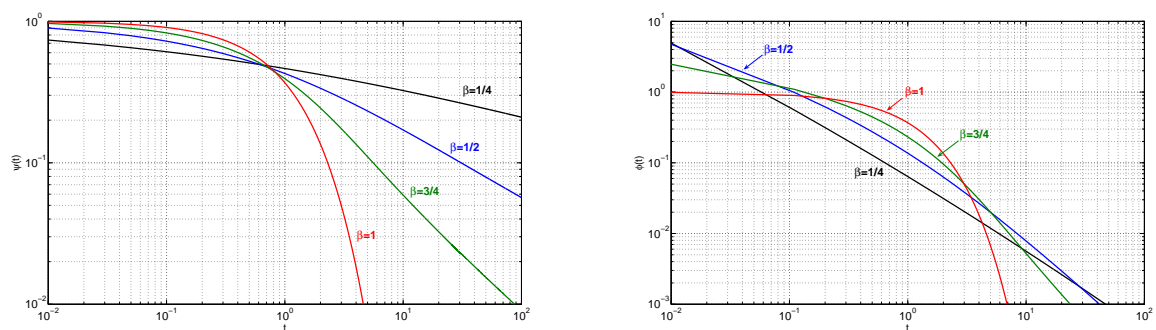

Fig. 2 The functions $\Psi(t)$ (left) and $\phi(t)$ (right) in logarithmic scales
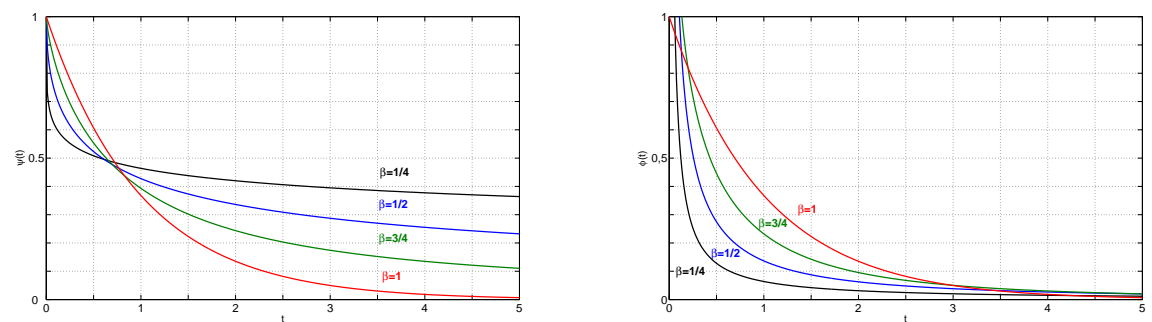

Fig. 3 The functions $\Psi(t)$ (left) and $\phi(t)$ (right) in linear scales 


\section{References}

[1] V. Balakrishnan, Anomalous diffusion in one dimension, Physica A 132 (1985), 569-580.

[2] E. Barkai and R.J. Silbey, Fractional Kramers equation, J. Phys. Chem. B 104 (2000), 3866-3874.

[3] E. Barkai and I.M. Sokolov On Hilfer's objection to the fractional time diffusion equation, Physica A 373 (2007), 231-236.

[4] M. Caputo, Linear models of dissipation whose $Q$ is almost frequency independent, Part II. Geophys. J. R. Astr. Soc. 13 (1967), 529-539.

[5] M. Caputo, Elasticità e Dissipazione. Bologna, Zanichelli (1969).

[6] M. Caputo and F. Mainardi, Linear models of in anelastic solids. Riv. Nuovo Cimento (Ser. II) 1 (1971), 161-198.

[7] D.R. Cox, Renewal Theory, 2-nd Edn., Methuen, London (1967).

[8] M.M. Dzherbashyan, Integral Transforms and Representations of Functions in the Complex Plane. Moscow, Nauka (1966). In Russian. [Note that there is also the transliteration as Djrbashyan]

[9] M.M. Dzherbashyan and A.D. Nersesian, Fractional derivatives and the Cauchy problem for differential equations of fractional order. Izv. Acad. Nauk Armjanskvy SSR, Matematika 3 (1968), 3-29. In Russian.

[10] A. Erdélyi, W. Magnus, F. Oberhettinger, and F.G. Tricomi, Higher Transcendental Functions, Vol. 3, McGraw-Hill, New York (1953-1954).

[11] W. Feller, On a generalization of Marcel Riesz' potentials and the semi-groups generated by them, Meddelanden Lunds Universitets Matematiska Seminarium (Comm. Sém. Mathém. Université de Lund). Tome suppl. dédié a M. Riesz, Lund, 1952, pp. 73-81.

[12] W. Feller, An Introduction to Probability Theory and its Applications, Vol. 2, 2-nd edn. Wiley, New York (1971). [1-st edn. 1966]

[13] I.M. Gel`fand and G.E. Shilov, Generalized Functions, Volume I. Academic Press, New York and London (1964). 
[14] B.V. Gnedenko and A.N. Kolmogorov, Limit Distributions for Sums of Independent Random Variables, Addison-Wesley, Cambridge, Mass.(1954). [Translated from the Russian edition, Moscow 1949, with notes by K.L. Chung, revised 1968]

[15] B.V. Gnedenko and I.N. Kovalenko, Introduction to Queueing Theory, Israel Program for Scientific Translations, Jerusalem (1968).

[16] R. Gorenflo and E. Abdel-Rehim, From power laws to fractional diffusion: the direct way, Vietnam Journal of Mathematics 32 SI (2004), 65-75.

[17] R. Gorenflo, Yu. Luchko, F. Mainardi, Analytical properties and applications of the Wright function, Fractional Calculus and Applied Analysis 2 (1999), 383-414. [E-print arXiv:math-ph/0701069

[18] R. Gorenflo, Yu. Luchko, F. Mainardi, Wright functions as scaleinvariant solutions of the diffusion-wave equation, J. Comput. Appl. Math. 118 (2000), 175-191.

[19] R. Gorenflo and F. Mainardi, Fractional calculus: integral and differential equations of fractional order. In A. Carpinteri and F. Mainardi (Editors), Fractals and Fractional Calculus in Continuum Mechanics, Springer Verlag, Wien and New York (1997), pp. 223-276. http://www.fracalmo.org

[20] R. Gorenflo and F. Mainardi, Random walk models for space fractional diffusion processes, Fractional Calculus and Applied Analysis 1 (1998), 167-191.

[21] R. Gorenflo and F. Mainardi, Fractional diffusion processes: probability distributions and continuous time random walk, in: G. Rangarajan and M. Ding (Editors), Processes with Long Range Correlations, SpringerVerlag, Berlin (2003), pp. 148-166. [Lecture Notes in Physics, No. 621]

[22] R Gorenflo and F. Mainardi, Simply and multiply scaled diffusion limits for continuous time random walks, in: S. Benkadda, X. Leoncini and G. Zaslavsky (Editors), Proceedings of the International Workshop on Chaotic Transport and Complexity in Fluids and Plasmas Carry Le Rouet (France) 20-25 June 2004, IOP (Institute of Physics) Journal of Physics: Conference Series 7 (2005), 1-16. 
[23] R. Gorenflo, F. Mainardi, E. Scalas and M. Raberto Fractional calculus and continuous-time finance III: the diffusion limit, in: M. Kohlmann and S. Tang (Editors), Mathematical Finance, Birkhäuser Verlag, Basel (2001), pp. 171-180.

[24] R. Gorenflo, F. Mainardi, H.M. Srivastava, Special functions in fractional relaxation-oscillation and fractional diffusion-wave phenomena, in: D. Bainov (Ed.), Proceedings VIII International Colloquium on Differential Equations, Plovdiv 1997 VSP, Utrecht, 1998, pp. 195-202.

[25] R. Gorenflo, F. Mainardi and A. Vivoli, Continuous time random walk and parametric subordination in fractional diffusion, Chaos, Solitons and Fractals 34 (2007), 87-103. [E-print arXiv:cond-mat/0701126]

[26] R. Gorenflo, A. Vivoli and F. Mainardi, Discrete and continuous random walk models for space-time fractional diffusion, Nonlinear Dynamics 38 (2004), 101-116.

[27] R. Hilfer, Exact solutions for a class of fractal time random walks, Fractals 3 (1995), 211-216.

[28] R. Hilfer, On fractional diffusion and continuous time random walks, Physica A 329 (2003), 35-39.

[29] R. Hilfer and L. Anton, Fractional master equations and fractal time random walks, Phys. Rev. E 51 (1995), R848-R851.

[30] T. Huillet, Renewal processes and the Hurst effect, J. Phys. A 35 (2002), 4395-4413.

[31] T. Huillet, On the waiting time paradox and related topics, Fractals 10 (2002), 1-20.

[32] V. Kiryakova, Generalized Fractional Calculus and Applications. Harlow, Longman (1994). [Pitman Research Notes in Mathematics, Vol. 301]

[33] A.N. Kochubei, A Cauchy problem for evolution equations of fractional order, Differential Equations 25 (1989), 967-974. [English translation from the Russian Journal Differentsial'nye Uravneniya]

[34] A.N. Kochubei, Fractional order diffusion, Differential Equations 26 (1990), 485-492. [English translation from the Russian Journal Differentsial'nye Uravneniya] 
[35] M. Kotulski, Asymptotic distributions of continuous-time random walks: a probabilistic approach, J. Stat. Phys. 81 (1995), 777-792.

[36] T.J. Kozubowski and S.T. Rachev, Univariate geometric stable laws, J. Comput. Anal. Appl. 1, (1999), 177-217.

[37] F. Mainardi, Fractional relaxation-oscillation and fractional diffusionwave phenomena. Chaos, Solitons and Fractals 7 (1996), 1461-1477.

[38] F. Mainardi, Fractional calculus: some basic problems in continuum and statistical mechanics, in A. Carpinteri and F. Mainardi (Editors), Fractals and Fractional Calculus in Continuum Mechanics. Wien and New York, Springer Verlag, 1997, pp. 291-348.

[39] F. Mainardi and R. Gorenflo, On Mittag-Leffler-type functions in fractional evolution processes, J. Comput. Appl. Math. 118 (2000), 283299.

[40] F. Mainardi, R. Gorenflo, E. Scalas, A fractional generalization of the Poisson processes. Vietnam Journal of Mathematics 32 SI (2004), 5364. [E-print arXiv:math-pr/0701454]

[41] F. Mainardi, R. Gorenflo and A. Vivoli, Renewal processes of MittagLeffler and Wright type, Fractional Calculus and Applied Analysis, 8 (2005), 7-38. [E-print arXiv:math-pr/0701455

[42] F. Mainardi, Yu. Luchko and G. Pagnini, The fundamental solution of the space-time fractional diffusion equation, Fractional Calculus and Applied Analysis 4 (2001), 153-192. [E-print arXiv: cond-mat/0702419]

[43] F. Mainardi and G. Pagnini, The Wright functions as solutions of the time fractional diffusion equations, Applied Mathematics and Computation 141 (2003), 51-62.

[44] F. Mainardi, M. Raberto, R. Gorenflo and E. Scalas, Fractional calculus and continuous-time finance II: the waiting time distribution, Physica A 287 (2000), 468-481.

[45] F. Mainardi, A. Vivoli and R. Gorenflo, Continuous time random walk and time fractional diffusion: a numerical comparison between the fundamental solutions, Fluctuation and Noise Letters 5 (2005), L291L297. 
[46] M.M. Meerschaert, D.A. Benson, H.-P. Scheffler and B. Baeumer, Stochastic solutions of space fractional diffusion equation, Phys. Rev. E 65 (2002), 041103-1/4.

[47] R. Metzler, E. Barkai and J. Klafter, Anomalous diffusion and relaxation close to thermal equilibrium: a fractional Fokker-Planck equation approach, Phys. Rev. Lett 82 (1999), 3563-3567.

[48] E.W. Montroll and H. Scher, Random walks on lattices, IV: Continuoustime walks and influence of absorbing boundaries, J. Stat. Phys. 9 (1973), 101-135.

[49] E.W. Montroll and G.H. Weiss, Random walks on lattices, II, J. Math. Phys. 6 (1965), 167-181.

[50] E.W. Montroll and D.J. West, On an enriched collection of stochastic processes, in E.W. Montroll and J. Leibowitz (Editors), Fluctuation Phenomena, North-Holland, Amsterdam, 1979, pp. 61-175. [Studies in Statistical Mechanics, Vol. VII]

[51] R.N. Pillai, On Mittag-Leffler functions and related distributions, Ann. Inst. Statist. Math. 42 (1990), 157-161.

[52] A. Piryatinska, A.I. Saichev and W.A. Woyczynski, Models of anomalous diffusion: the subdiffusive case, Physica A 349, 375-420 (2005).

[53] I. Podlubny, Fractional Differential Equations. San Diego, Academic Press (1999).

[54] A. Renyi, A characteristic of the Poisson stream, Proc. Math. Inst. Hungarica Acad. Sci. 1 (4), 563-570 (1956). [In Hungarian]

[55] S.M. Ross, Introduction to Probability Models, 6-th Edn. New York, Academic Press (1997).

[56] A.I. Saichev and G.M. Zaslavsky, Fractional kinetic equations: solutions and applications, Chaos 7 (1997), 753-764.

[57] S.G. Samko, A.A. Kilbas and O.I. Marichev, Fractional Integrals and Derivatives: Theory and Applications, Gordon and Breach, New York (1993). Translation from the Russian edition, Nauka i Tekhnika, Minsk (1987). 
[58] E. Scalas, The application of continuous-time random walks in finance and economics, Physica A 362 (2006), 225-239.

[59] E. Scalas, R. Gorenflo and F. Mainardi, Fractional calculus and continuous-time finance, Physica A 284 (2000), 376-384.

[60] E. Scalas, R. Gorenflo and F. Mainardi, Uncoupled continuous-time random walks: Solution and limiting behavior of the master equation, Phys. Rev. E 69 (2004), 011107-1/8.

[61] W.R. Schneider and W. Wyss, Fractional diffusion and wave equations, J. Math. Phys. 30 (1989) 134-144.

[62] I.M. Sokolov, J. Klafter and A. Blumen, Do strange kinetics imply unusual thermodynamics? Phys. Rev. E. 64 (2001), 021107/1-4.

[63] T. Szàntai, Limiting distribution for the sums of random number of random variables concerning the rarefaction of recurrent events. Studia Scientiarum Mathematicarum Hungarica 6 (1971), 443-452.

[64] T. Szàntai, On an invariance problem related to different rarefactions of recurrent events. Studia Scientiarum Mathematicarum Hungarica 6 (1971), 453-456.

[65] V.V. Uchaikin and V.V. Saenko, Stochastic solution of partial differential equations of fractional orders, Siberian Journal of Numerical Mathematics 6 (2003), 197-203.

[66] G.H. Weiss, Aspects and Applications of Random Walks, NorthHolland, Amsterdam (1994).

[67] K. Weron and M. Kotulski, On the Cole-Cole relaxation function and related Mittag-Leffler distribution, Physica A 232 (1995), 180-188.

[68] D.V. Widder, The Laplace Transform, Princeton University Press, Princeton (1946). 